\title{
Prediction of Baby Emotions through Pattern Recognition by Monitoring Baby's Biological Measurements
}

\author{
Vethapriyan Kugamoorthy*, Dinithi Nallaperuma \\ Informatics Institute of Technology, 57, Ramakrishna Road, Colombo, Sri Lanka. \\ * Corresponding author. Tel.: +94776492262; email: priyan1994@gmail.com \\ Manuscript submitted December 19, 2015; accepted May 8, 2016. \\ doi: $10.17706 /$ jcp.12.5.381-388
}

\begin{abstract}
The world humans live in today is a victim of rapid changes due to the high evolvement in science and technology. Baby Monitoring have turned into a major problem as it can cause significant issues in both parents and infants. Because parents have to occupy in some profession in order to cover the expenditures infants are left by at home alone, which makes parents anxiety which will affect the kids. According to research statistics 2300 babies still die yearly in the USA.

Researchers and developers have discovered and implemented solutions for this which has not addressed the problem completely. Studies show that the reason for this is the limitations in technology, fewer acceptances from the public and being expensive and too complicated to implement.

Main challenge in solving this issue is to build a solution which is accurate, easy to use, and cost effective and will be accepted by the public. Therefore, a baby monitor product (Care) has been proposed. View babies status, Send or receive audio, get notify if any abnormalities occur and generate reports are the main features this solution will consist. The product consist four main parts such as baby's wearable device, smartphone application, web server and audio server. Emotions plays major role in the growth of babies. Interest, distress, disgust, smile, anger, sadness, surprise and etc. are some emotions practiced by babies. When baby cries for long period it will affect the mental growth, so the care should be concerned more with each emotions.
\end{abstract}

Key words: Parents, infants, baby monitor, emotions.

\section{Introduction}

Once a couple is meant to be together the next important step of their life together is to have an offspring, which in human terms people called the baby. Babies or infants are extremely lovable and parents are the caretaker or the guardians of them, who show unconditional love towards their offspring.

Since then the story has been changed to a great extent. The world humans live in today is a victim of rapid changes due to the high evolvement in science and technology. Even though the Earth takes 24 hours to rotate itself on its own curvature, modern people find it harder to be cooperative with their own work in a day. People have more work than any other days in the past due to increased needs. More money is a necessity these days, so humans work very hard and in this modern business environment people act in competitive manner. The idea to have a very strong family unit is fading these days.

Still the idea of having a baby makes bonds very stronger in a family. Yet nowadays new parents find it very hard to find extra time to be with their babies, cuddle their babies. The number of working mothers of 
dependent babies has been increased up to 54 percent in America along, among all mothers by 1992 [1]. Across the Atlantic in the UK the same figure has been increased to 72 percent in 2013 [2]. Therefore, it shows that the infants are left behind alone or may be with a stranger who humans called as a caretaker, who might not very familiar with the baby and their activities.

Parents' involvement is an utmost impotency for a child to build their character. It is shown that more than 2300 babies still die annually in the USA only because of sudden causes and lack of care. Moreover, Powell [3] has shown that lack of baby observance could cause baby suffocation or strangulation and reports shows that the number of both suffocation and strangulation has been increased from 2.8 to 12.5 deaths per 100,000 babies during 1984 to 2004 .

\section{Related Work}

In this 21st century there are lots of baby monitors out in market. The Evolution of Baby Monitoring systems is like, first humans had Audio Baby Monitors which allows users to listen to their babies later they got Video Monitors where users are possible to listen and view the baby in live and Smart Diaper which will informing the parents' mobile phones when it is require to change the diaper with the chemical composition of the babys' urine. Now humans got Wearable Baby monitors which allow users to get information about babys' biological data.

\subsection{Audio Baby Monitors}

The Audio Baby Monitor (Baby Alarm) is a system which uses radio signals to keep track of the infant's movements from a distance. This system has two ends which are the transmitter and the receiver. These two ends communicate via radio signals. The transmitter unit is equipped with microphones while placing near to the baby and the other end will be near the parent which is equipped with speakers. Although, some systems are equipped with two way communication systems which mean both ends have both speakers and microphones, which allow the parents to play music or to speak to the infant [4].

Nevertheless, earlier systems have few complications like baby strangulations from cords of the system etc. These problems have leaded the parents to use video monitors along with audio monitors.

\subsection{Video Monitors}

Video monitors have the same technology behind, like the audio monitors described, above. The only difference among those systems is that, this system uses video feedback from the transmitter about the baby and makes it possible for the parents to see what is happening with the baby in live pictures. So the transmitter is equipped both with a camera and a microphone and the receiver is equipped with display units and speakers mainly.

Unfortunately, when it is dark or when it is night the video is useless since you cannot see anything from normal video recording because the normal cameras cannot capture pictures in dark. Therefore you need special recording cameras such as infra-red cameras. That camera catches the baby movements in the dark as well [5].

\subsection{Wearable Monitors}

Main limitation in the above mentioned monitors was cannot monitor babys' biological data in real time. This limitation made developers and researchers to come up with Wearable Monitors. Basically these monitors will have a device which will be worn by babies. This device made with wearable sensors which will gather physiological and movement data.

From the above mention device using wireless data transmission techniques gathered data will be send to parents mobile phone. Using Smartphone applications parents are possible to view their babies' status in real 
time.

The most important feature is convenient use due to its expedient design. Also portable parent unit allows the parents to monitor baby's movement and they can check the temperature of baby's' environment through the unit by placing the device in baby's room which consist of inbuilt microphone and temperature sensor [6].

\section{Relationship between Emotion and Biological Data}

Even though humans having all kinds of baby monitoring solutions parents think there are something missing and having a feel of not satisfied. Wallace [7] says it is good to monitor babies' health status every day also Wallace [7] points out it is important to monitor babies' health status all the time in the early developmental stage of the baby's life because of SIDS . Linda Rodriguez McRobbie [8] have mention that the current baby monitoring devices which are in market even they monitor babies' health status they are not giving relief to parents and also McRobbie [8] mentioned as the companies are selling fear in the name of baby care systems because they alert constantly of tiny changes which will increase anxiety of parents.

\section{Solution}

Considering all the limitations and solutions mentioned above, a system which will track the biological data of infants and store in a database where pediatricians can access without showing to parents but alerts parents in bad situations and inform the babies' emotions which can be understand by humans will solve above mentioned requirements.

\subsection{Technologies}

The advanced feature mentioned in the solution is predicting the infants' emotion using infants' biological data. The quandary of emotion detection predicated on the quantified biological transmutations in the human body had received an important attention lately.

In the past few decades, many studies have been done on emotion recognition. Anderson and McOwan [9] utilized visages to recognize emotion. Ang and colleagues [10] did emotion recognition based on prosody. All the same, these signals shared the same disadvantage. They are not true to detect emotion, particularly when people desire to hide their feelings. Other signs were introduced into emotion recognition research in order to sweep over this disadvantage. Picard et al (2002) [11] used peripheral neurons system including heart rate and respiration to do this work. According to Nie et al (2001), in recent years, more and more researchers have started to use heart rate signals in recognizing emotion because they are reliable. However, the classification results are often not good enough.

Therefore, to provide this advanced feature, system needs to deal with a database which has biological data (Heart Rate and Temperature) and emotion. Moreover, system needs a method to predict emotion from these data also needs to direct the machine to learn these data to examine the relationship between these biological data and emotion. Therefore, the following major research areas are identified.

- Data Mining

- Machine Learning

\subsection{Data Mining}

Data mining is a powerful new technology with great potential to help any industry. Like people say "Learn from History" industry can learn from these data. Basic idea of Data mining is predict behaviors and future trends.

The human emotional status is rather intangible, and therefore cannot be instantly quantified. However, these emotions can be correlated to external and/or internal factors, which are rather tangible things, and 
hence they can be quantified and analyzed. The internal factors come from different regions of the physical structure in various configurations such as heart rate (HR), heart rate variability (HRV), electroencephalography (EEG), respiration rate (RR), finger temperature (FT), and others.

These factors' measurements are provided in wide ranges and often their impacts vary from a person to a person and for different postures for the same individual. For instance, a given measurement of some factors may pertain to a person being happy, while the same measurements may reveal a rather "sad" status of some other individual. This sort of behavior lends itself naturally to Rule based model (zero and one or true and false).

\section{Rule Based}

A rule based system consists of if-then rules, a bunch of facts, and an interpreter controlling the application of the rules. According to Khanna (2013) [12], one of the major strength of rule based representation is its ability to represent various uncertainties. Uncertainty is inherently part of most human decision making. This uncertainty could arise from various sources like incomplete data or domain knowledge used being unreliable [12].

Khanna and Sasikumar (2013) [12], says to handle these uncertainties, there are two broad approaches those representing uncertainty using numerical quantities and those using symbolic methods.

Approaches to handle uncertainties,

1) Numerical approaches

These approaches are useful for managing the issues pertained to unreliable or inaccurate knowledge. Numerical approaches

- Bayesian reasoning.

- Evidence theory.

- Fuzzy set approaches.

2) Symbolic approaches

These approaches are mostly aimed at handling incomplete information.

Symbolic approaches

- Assumption Based Reasoning.

- Default Reasoning.

- Non-monotonic Logic.

For instance, if there is not enough data available, by using Symbolic approach the system makes assumptions that can be corrected later, when more data is obtained. In this system, as it is focused on babys' emotion having huge database will be hard additionally the basic problem is that there are hardly any variables or variable combinations which can infer any emotion to complete certainty. Therefore, to handle the uncertainty numerical approaches concentrated also Fuzzy set numerical models analysed.

\section{Fuzzy Logic}

Fuzzy logic is an approach to computing based on "degrees of truth" rather than the usual "true or false" (1 or 0) Boolean logic on which the modern computer is based. FL incorporates a simple, rule-based IF X AND Y THEN Z approach to a solving control problem rather than attempting to model a system mathematically.

Example:

IF temperature IS very cold THEN stop fan, IF temperature IS hot THEN speed up fan

Therefore, by using fuzzy operations system will interpret the knowledge about each component. Moreover, this will enable the system to discover the emotion of a baby using fuzzy inputs of the assorted components. For example, system can use a fuzzy rule such as "IF (Temperature is Low) AND (Heart Rate is Low) THEN (Baby is Sad)". 


\subsection{Machine Learning}

Machine Learning algorithms are used to train the system to learn the relationships by using data. For example, a machine learning system could be trained on the body temperature data to learn to distinguish between Celsius data and Fahrenheit data. After learning, it can then be used to classify new data. Machine learning algorithms can be organized into a taxonomy based on the desired outcome of the algorithm or the type of input available during training the machine.

Learning algorithms can be categorize as

1) Supervised learning

Training samples used to design were labelled by their category membership.

2) Unsupervised learning

Procedures work with unlabelled samples but unsupervised learning has advantages over supervised learning.

- Labelling a large number of samples is expensive and time consuming.

- Characteristics of the pattern can change slowly over time. These changes cannot track over time in an unsupervised mode.

- Gain some insight into the nature or structure of data.

Even though machine learning is well known among developers similar systems rarely uses machine learning algorithms because of this the result accuracy they getting is also low and few systems have failed too. Therefore, by using the machine learning algorithms system can increases its accuracy of predicting. For this project Artificial Neural Network is being analysed as it is considered well known unsupervised machine learning approach among developers also it has been used in similar predicting systems and gave successful results.

\section{Artificial Neural Network}

Artificial Neural Networks are comparatively simple electronic models based on the neural structure of the brain. The brain basically learns from experience likewise ANN makes system learns from the past inputs and outputs and gives outputs (e.g. a child learns to recognize dogs from examples of dogs).

Even though fuzzy sets and operations are useful for representing the information base, they fail to model the individual deportment of each and every individual. Therefore, an adaptive learning mechanism is needed to correct the model if we were to provide for the differences in emotions between various babies. According to Malkawi [13], "The ANN model does not allow the use of fuzzy sets or rules". Moreover, Malkawi [13] mentioned to use the benefits of both fuzzy logic and artificial neural networks, system can use the hybrid method, which combines fuzzy logic and artificial neural networks in a single model.

\section{Requirements from Parents}

The primary target groups of Care are parents who would wish to make the best for their kids. With the growth in the cost of living, the number of working mothers has grown exponentially. Thus, a solution to give relief to working mothers and draw the emotional relationship between parents and infants, even if they didn't spend more time together. As there is wide range of parents Questionnaires selected as requirement gathering methodology.

An online survey was published in order to gather more details mainly targeted for the working parents and the domain experts who are the main stakeholders of 'Care'. The results were gathered from 49 responses over a period of two weeks. Out of the 49 parents, $70 \%$ of parents are working. Thus, these mothers could express themselves better as they had been separated from their babies due to their long hours of work. The recruited mothers were between the age group of 20 to 25 with 28 of them between 26 to 30 years of age, 8 and the rest 31 and above years of age. 


\subsection{Findings}

It is obvious for the working mothers to worry about juggling jobs and family responsibilities. They feel guilty about jeopardizing their baby's emotional and social growth by going to work. By asking how many hours per day parents spend away from baby (Fig. 1) and how they feel when they are away from baby (Fig. 2) it's clear that more than $60 \%$ of parents stay away from baby for more than 8 hours per day also $100 \%$ of parents not felling happy when they are away from their parents. Moreover, $50 \%$ of babies are taking care by unknown people while parents are away while the rest $50 \%$ of babies taking care by his/her grandparents then the parents are less worried about them as compared to the ones who leave their babies with unknown people (maid or baby care centers).

How many hours do you spend away from your kid(s) per day?

How you feel when you are away from your kid(s)?

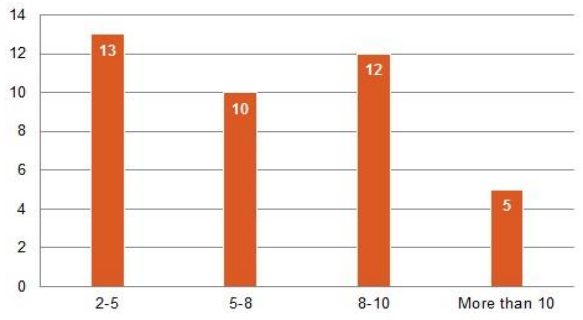

Fig. 1. Overview of how parents stay away from child(ren).

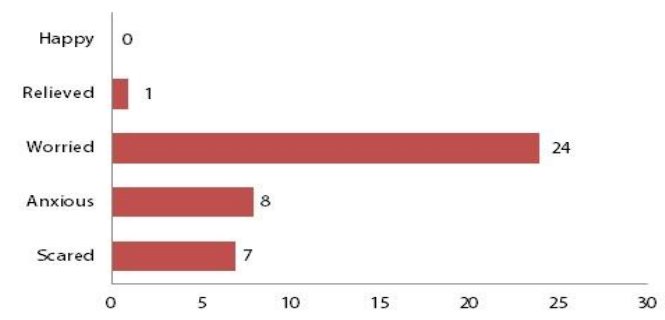

Fig. 2. How parents feel when they are away from $\operatorname{kid}(s)$.

\section{Design Implication}

After the analysis of the data, a systematic approach has been proposed to design and develop a biological signal monitoring wearable device which captures temperature, heart rate, physical activity, position. These data would be processed to interpret the baby's emotion. This non-clinical system would inform the parents about the emotion of the baby which would allow the mother to respond accordingly with two way communications. Moreover, parents will get notify if any abnormalities occur and parents can generate reports. Below Fig. 3 is high level design.

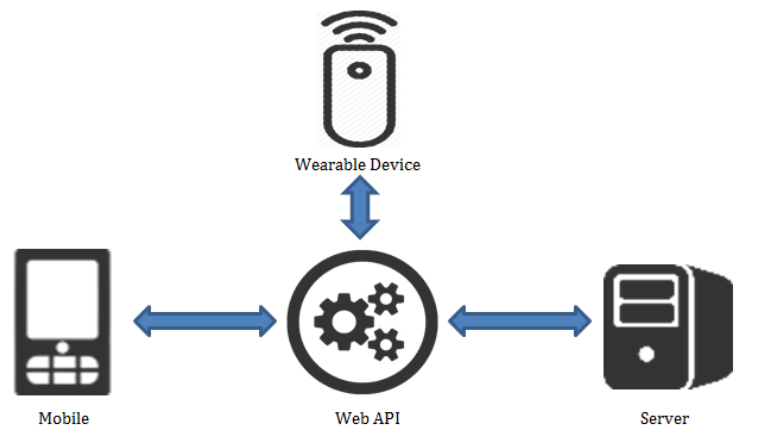

Fig. 3. High level design.

Analysis of related work study was used to design the hardware system. Most of the existing wearable monitors devices are attached to the babys' wrist. Meanwhile, some monitors devices attached to the cloth of the baby, so that it does not make the baby uncomfortable yet if the sensors attached in cloths contact with body will get disconnected frequently. Therefore, the proposed system would consist of a wrist band which consists of sensors to extract the babys' heart rate, temperature and position. The interpretation of the baby's status would be done by an expert system which would be based on the data collected from the baby and the data stored in the central database. 
Another question of how often they would want to receive information about the baby and how they would want to receive the information, from the responses, it's clearly shown that approximately $45 \%$ of the parents want to get information every 30 minutes, yet rest $55 \%$ of the parents are needed in different time period. Therefore, as a default settings system will set every 30 minutes to notify the parents also by giving access to modify this setting as they wish will increase the user friendliness of the system. According to the answers $87 \%$ of parents prefer using text and graphic as the main ways of output. Therefore if any abnormalities occur parents will get notify by SMS with the use of SMS gateway service. On the other hand, health status data would be saved in a central database which would be used for generate reports.

\section{Conclusion}

The parents' presence and role in the early developmental stage of the baby's life is very important. Though, many modern working parents find hard in balancing career with spending personal time with their baby due to the increased needs. This paper analyzed the effects of disseverment of working parents from their babies predicated on the survey conducted with 49 parents around the world. The outcomes of the study fortifies the above mentioned issue by verbalizing that most of the parents worry about their infants and spend quite a plethora of time cerebrating about infants while they are away from them. The paper withal proposes a system to develop a wearable monitoring system which captures biological data of the baby to predict the emotional status. The analysis of the survey showed that parents would optate to ken about their babys' emotions and health status while they are away from him/her. They would also like to communicate with their baby whenever they feels like and would feel happy and connected utilizing a device which would support them do that. Therefore, the proposed system would be a two way communication system through which the information about the baby would be given to the parents and the baby would also feel connected to his/her parents. This would benefit the working parents as it would make them feel relieved and assured which would not only avail concentrate better on their work, but also connect more with their baby while they are away. As a result, the paper concludes that technology would absolutely help working parents enhance their emotional connection with their baby.

\section{References}

[1] Novello, A. C. (1992). Parents Speak Out for America's Children. Conference Report. United States: Public Health Service.

[2] Women in the labour market. (2013). United Kingdom: Office for National Statistics.

[3] Powell, K. (2013). SIDS rate has declined, but 2,300 U.S. babies still die annually. From http://www.washingtonpost.com/national/health-science/sids-rate-has-declined-but-2300-us-babies -still-die-annually/2013/08/05/56dffca0-f0b6-11e2-9008-61e94a7ea20d_story.html

[4] Ray, L. How Does a Baby Monitor Work. http://www.ehow.com/how-does_4728283_baby-monitor-work.html

[5] Spurrier, R. (2014). How to Choose the Best Video Monitor for You and Baby.

[6] Press, A. (2013). Angelcare Baby Monitors.

[7] Wallace, K. (2014). Parents should read aloud to infants every day, pediatricians say. From http://edition.cnn.com/2014/06/25/living/doctors-read-aloud-to-infants-parents/index.html

[8] McRobbie, L. R. (2014). Selling Fear.

[9] Anderson, K, \& McOwan, P. W. (2006). A real-time automated system for the recognition of human facial expressions. IEEE Trans Syst Man Cybern B Cybern, 36(1), 96-105.

[10] Ang, J., Dhillon, R., Krupski, A., Shriberg, E., \& Stol, A. (2002). Prosody-based automatic detection of annoyance and frustration in human-computer dialog. Proceedings of Eighth Int. Conf. Spoken Language 
Processing (pp. 2037-2039).

[11] Picard, R. W., Vyzas, E., \& Healey, J. (2002). Toward machine emotional intelligence: Analysis of affective physiological state. IEEE, 23(10), 1175-1191.

[12] Khanna, P. S. (2013). Rule based system for recognizing emotions using multimodal approach. International Journal of Advanced Computer Science and Applications, 4(7), 32-39.

[13] Malkawi, M., \& Omayya, M. (2013). Artificial neuro fuzzy logic system for detecting human emotions. Human-Centric Computing and Information Sciences, 3(1), 1-13.

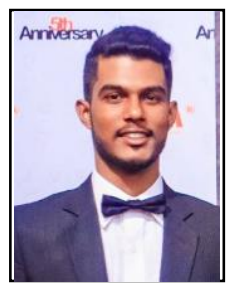

Vethapriyan Kugamoorthy was born at Jaffna Sri Lanka on 9 June, 1994. His primary studies were carried at Royal College Colombo from 2000 to 2010. Afterwards for the foundation studies, this is prior to higher studies attended at Informatics Institute of Technology (IIT) on May 2011 to Dec. 2011. After finishing the foundation course, he continued with the diploma and the degree of BEng (Hons) in software engineering at IIT in collaboration with University of Westminster in London UK from Jan. 2012 to Jun. 2015 respectively and graduated in 2015 with first class.

During the degree programme, he joined with Inova IT Systems Pvt Ltd at Colombo as an intern to get the initial working experience as a trainee software engineer from Jun. 2013 to Jun 2014. Then again he joined and currently works at the same company as a software engineer after the finishing the degree.

Mr. Kugamoorthy is an active member of Rotaract Club at IIT and awarded as the best Rotaractor of the month on May 2014. He had the privilege to work as the community service director for the club in year 2012/2013. During this time several charity events were organized. Meanwhile had the opportunity to be elected as the vice president in 2013/2014. During the vice presidency, he planned two fund raising events to sponsor the cancer hospital Maharagama Colombo. "Miracle" is the first to be staged, which is a controversial modern magic show, which was followed by a hot dog eating competition named as "Hot Dog Show Down". "Miracle" was awarded the gold as the most outstanding fund raising initiative and "Hot Dog Showdown" was also awarded with the bronze as the most outstanding club service project of the year in 2014/2015 by the Rotaract district committee at 24th Rotaract district assembly.

Dinithi Nallaperuma received the B.Sc. (Hons) in software engineering in 2010 and M.Sc. in advanced software engineering in 2015 from University of Westminster, UK. She is currently pursuing the $\mathrm{PhD}$ in data analytics at La Trobe University, Australia.

After the completion of the degree, Dinithi was a software engineer in Sri Lanka. From 2011 to 2013, Dinithi was a research engineer at National University of Singapore. Since 2013 she has been an assistant lecturer at Informatics Institute of Technology, Sri Lanka. 